\title{
A Compact Slot Antenna Utilizing a Right/Left-Handed Transmission Line Feed
}

\author{
Yu-Shin Wang, Min-Feng Hsu, and Shyh-Jong Chung, Senior Member, IEEE
}

\begin{abstract}
A novel compact planar antenna utilizing cascaded right/left-handed transmission lines is proposed. The significance of layout planning with respect to radiation is investigated and discussed in this paper. Two segments of transmission lines of the same electrical length with opposite signs are cascaded to provide zero phase at the operation frequency. The closed-form formulas for the equivalent circuit of a transmission line are used for circuit design. Both antenna layout and radiation mechanism are discussed. For experimental demonstration, the proposed antenna operating at $2.45 \mathrm{GHz}$ was implemented on an FR4 substrate. The topology using several patches has quite smaller size than the conventional patch antenna. Besides, a fairly omnidirectional radiation pattern was measured.
\end{abstract}

Index Terms-Antenna layout, left-handed (LH) transmission line, metamaterial, printed antenna, radiating edge.

\section{INTRODUCTION}

M ETAMATERIALS have recently been extensively discussed and studied. This kind of artificial structure has been utilized for many guided and unguided wave applications. Their unique characteristics have been considered to be valuable topics. Specifically, left-handed materials (LHMs) possessing negative refractive index have drawn tremendous interests in both scientific and engineering fields. The new concept associated with LHMs has led to the development of materials and structures with unprecedented properties. In particular, it contributes to the antenna design of either the radiator itself [1]-[19] or the feeding structure [20]-[26]. Metamaterial antennas now are popular research topics.

The conventional right-handed transmission line (RH TL) features the equivalent circuit (EQC) model consisting of series per-unit-length inductances and shunt per-unit-length capacitances. The analogous topology of series per-unit-length capacitances and shunt per-unit-length inductances indicates left-handed transmission lines (LH TL). Along the power traveling direction, LH TL is characterized by the phase advance whereas RH TL is characterized by the phase delay. RH TL and LH TL can be embedded into each other and was named as composite right/left-handed transmission line (CRLH TL) [27], which actually provides an idea to insert LH TL into the

Manuscript received September 7, 2006; revised September 17, 2007. This work was supported in part by the National Science Council, R.O.C., under Contract NSC 96-2752-E-009-003-PAE.

The authors are with the Department of Communication Engineering, National Chiao Tung University, Hsinchu, Taiwan 30050, R.O.C. (e-mail: sjchung@cm.nctu.edu.tw).

Color versions of one or more of the figures in this paper are available online at http://ieeexplore.ieee.org.

Digital Object Identifier 10.1109/TAP.2008.916910

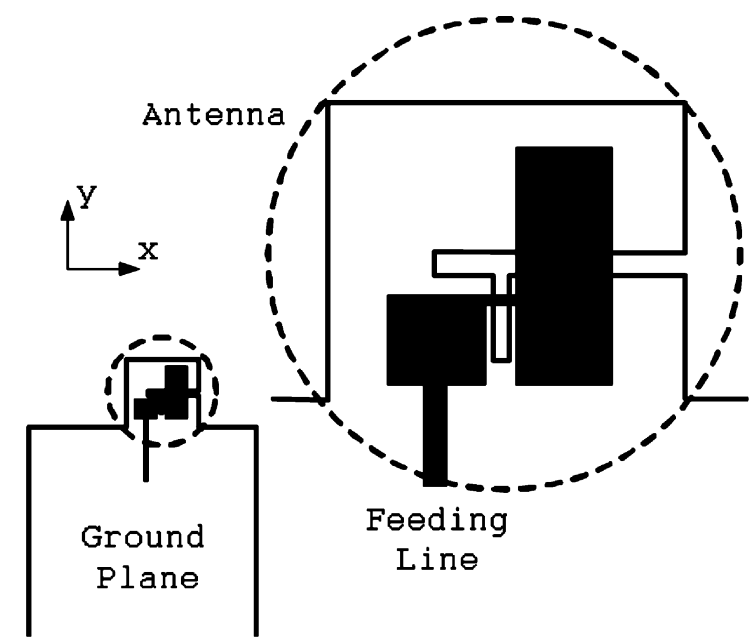

Fig. 1. Geometry of the proposed planar antenna.

host RH TL. The zeroth-order resonance (ZOR) [28] makes use of the opposite phase properties of RH and LH TL and has been proved experimentally. Small planar antennas utilizing the ZOR structure were published [12]-[14]. The physical size of such antenna can be arbitrary since it is specified by the value of the capacitances and inductances instead of the wavelength. The concept of the infinite wavelength resonant antenna was first demonstrated in [12]. However, it has relatively less compact size including the matching structure. Another published metamaterial antenna [13] consists of two substrate layers with one of which requiring quite small thickness and higher dielectric constant. The capability of dual-mode operation at two different frequencies was also presented in [14]. Other compact planar antennas using LH TL with periodically L-C loaded lines [10], [16] were presented. The investigated structures also demonstrated the possibility of reducing the physical size of microstrip patch antennas. For all the recent published antennas above, patch or patch-like radiation patterns were obtained. There are, however, scarce focused topics on physical structure and radiation mechanism for the small antennas. This paper intends to show the importance of the layout and the investigation for radiation.

Rather than employing CRLH or simply LH TL, a novel planar antenna utilizing cascaded right/left-handed transmission lines is proposed as sketched in Fig. 1. Two cascaded transmission lines of equal amount of electrical length with opposite polarities, i.e., a segment of RH TL and a segment of $\mathrm{LH}$ TL, can also result in phase of zero degree as shown in Fig. 2. With using the equivalent transmission line circuits, one can realize compact antennas consisting of printed inductors (L) 


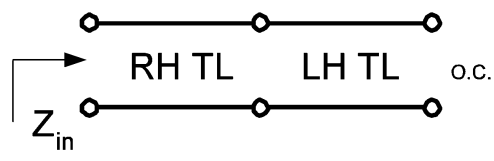

Fig. 2. Cascaded right/left-handed transmission lines.

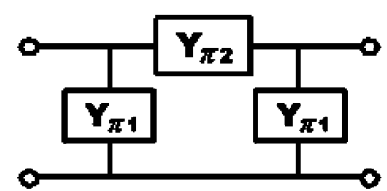

(a)

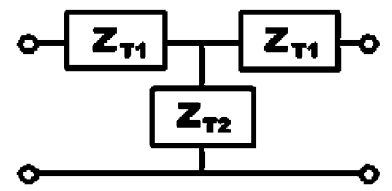

(b)
Fig. 3. (a) $\pi$ and (b) T EQC for a reciprocal two-port network.

and capacitors $(\mathrm{C})$ on a printed circuit board without via. The physical dimensions of the antenna are decided by the values of the inductances and capacitances. The layout planning has significant influences on the implementation of such radiating structures.

This paper is organized as follows. Section II presents the equivalent transmission line model. Section III presents the design of the proposed antenna and compares different layouts. Section IV discusses the radiation mechanism by investigating the simulation results. Further discussions and experiments are shown in Section V. Finally, Section VI concludes this study and draws some possible future works.

\section{EQUivalent TRANSMiSSION LiNE MODEL}

The design starts with cascading a segment of RH TL and a segment of LH TL. The former one has a positive electrical length indicating phase delay whereas the latter one has a negative electrical length indicating phase advance. One port is opencircuited as illustrated in Fig. 2. The input impedance $\left(Z_{\text {in }}\right)$ possesses zero imaginary part, which is one of basic requirements for a resonant antenna. By employing the EQC model for a TL segment, one can realize it with lumped circuits. With the specified characteristic impedance $\mathrm{Z}_{0}$, the operation frequency $f_{0}$, and the electrical length $\theta$, it is well-known that a certain length of transmission line can be modeled by either $\pi$ or T EQC. Fig. 3 shows the circuit models with

$$
\begin{aligned}
\mathrm{Y}_{\pi 1} & =j \mathrm{Y}_{0}(\csc \theta-\cot \theta) \\
\mathrm{Y}_{\pi 2} & =-j \mathrm{Y}_{0} \csc \theta \\
\mathrm{Z}_{T 1} & =j \mathrm{Z}_{0}(\csc \theta-\cot \theta) \\
\mathrm{Z}_{T 2} & =-j \mathrm{Z}_{0} \csc \theta
\end{aligned}
$$

where $Y_{0}=1 / Z_{0}$. These formulas can be easily derived by comparing the ABCD matrix for TL segment with the ABCD matrix for $\pi$ and T circuits at the design frequency [30]. The susceptances of $\mathrm{Y}_{\pi 1}, \mathrm{Y}_{\pi 2}$ and the reactances of $Z_{T 1}, Z_{T 2}$, as functions of the electrical length $\theta$, for the normalized component values with $\mathrm{Y}_{0}=1$ and $\mathrm{Z}_{0}=1$ are sketched in Fig. 4 . The upper half plane in Fig. 4(a) and the lower plane in Fig. 4(b) indicate capacitive elements whereas the lower half plane in Fig. 4(a) and the upper plane in Fig. 4(b) indicate inductive elements.

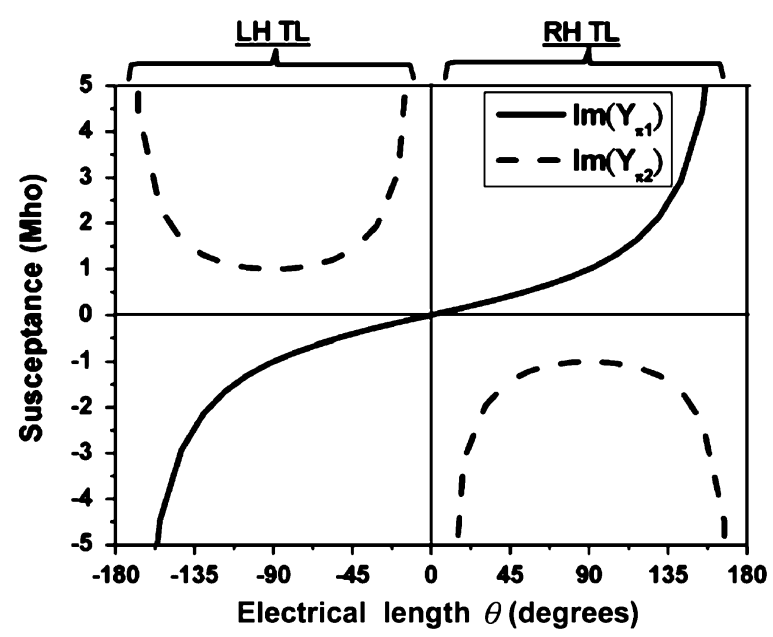

(a)

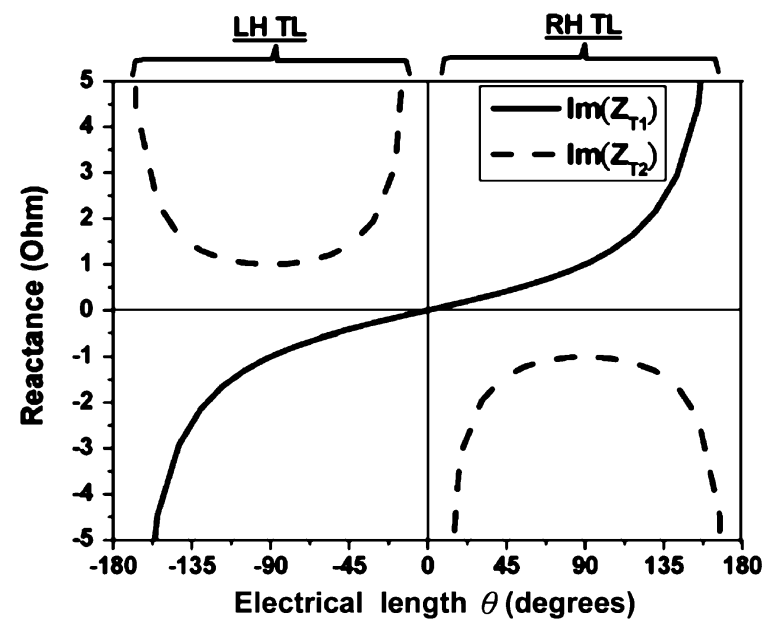

(b)

Fig. 4. (a) Susceptances of $Y_{\pi 1}, Y_{\pi 2}$ and (b) reactances of $Z_{T 1}, Z_{T 2}$, as functions of the electrical length $\theta$, for the equivalent circuits of Fig. 3. $\mathrm{Y}_{0}=1$ and $\mathrm{Z}_{0}=1$.

\section{DESIGN AND LAYOUT}

\section{A. Design of Circuit Parameters}

Following the previous section, formulas for the inductors (L) and capacitors $(\mathrm{C})$ of the equivalent transmission line model are listed in Table I, where $\omega_{0}=2 \pi f_{0}$. The notation $\mathrm{L}_{\mathrm{L} \pi}$, for example, represents the value of the inductor of the $\pi$ model for LH TL. Since each TL segment in Fig. 2 can employ either $\pi$ or $\mathrm{T}$ circuit, there are four combinations in total. A $\pi$ circuit for RH TL and a T circuit for LH TL are chosen and connected with an open-circuit at the unconnected port of the LH TL. This can results in a via-free layout which does not require an extra fabrication process. The last series capacitor of the $\mathrm{T}$ circuit can be removed since it is connected to the open-circuit end. As shown in Fig. 5, it turns out the circuit for designing the proposed antenna with $\mathrm{C}_{1}=\mathrm{C}_{2}=\mathrm{C}_{\mathrm{R} \pi}, \mathrm{L}_{1}=\mathrm{L}_{\mathrm{R} \pi}, \mathrm{C}_{3}=$ $\mathrm{C}_{\mathrm{LT}}$, and $\mathrm{L}_{2}=\mathrm{L}_{\mathrm{LT}}$.

In order to have a compact structure, the design of the circuit parameters was considered. Referring to Fig. 4, choosing $\theta$ close to either 0 or 180 degrees leads to very large $\mathrm{L}$ or $\mathrm{C}$ value. Therefore, $\theta$ is chosen to be 90 degrees so as to keep a small 


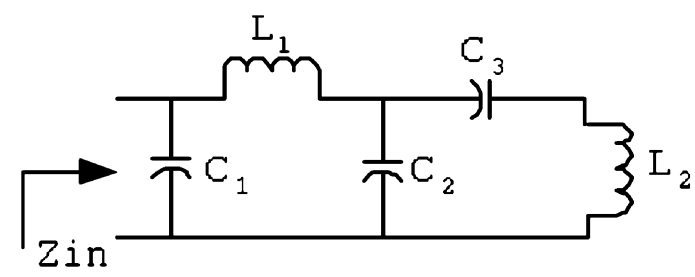

Fig. 5. Cascaded circuit structure consisting of a $\pi$ model for RH TL and a T model for LH TL. The last series capacitor of the T circuit has been removed since it is connected to the open-circuit end.

TABLE I

FORMULAS OF L AND C FOR A TL SEGMENT

\begin{tabular}{|c|c|c|}
\hline BQC & Formules for $\mathrm{L}$ & Formulas for C \\
\hline$\pi_{\text {LH }}$ & $L_{L r}=\frac{Z_{0}}{(\csc \theta-\cot \theta) \alpha_{0}}$ & $C_{L r}=\frac{1}{Z_{0} \omega_{0} \sin \theta}$ \\
\hline$\pi_{\mathrm{RH}}$ & $\mathrm{L}_{R z}=\frac{Z_{0} \sin \theta}{\omega_{0}}$ & $C_{R \theta}=\frac{(\csc \theta-\cot \theta)}{Z_{0} \mu_{0}}$ \\
\hline$T_{L H}$ & $L_{L T}=\frac{Z_{0}}{\omega_{0} \sin \theta}$ & $C_{L I}=\frac{1}{Z_{0} \alpha_{\theta}(\csc \theta-\cot \theta)}$ \\
\hline $\mathrm{T}_{\mathrm{RH}}$ & $\mathrm{L}_{R \sigma}=\frac{Z_{0}(\csc \theta-\cot \theta)}{a_{0}}$ & $\mathrm{C}_{R T}=\frac{\sin \theta}{Z_{0} a_{0}}$ \\
\hline
\end{tabular}

circuit area. In addition, since many published antennas using metamaterial concepts, mentioned in Section I, possess patch or patch-like radiation patterns, this study goes with introducing relatively larger capacitors rather than larger inductors. In case of avoiding large inductors and mainly having patches in the physical structure, a relatively small $Z_{0}$ is preferred. Based on these conditions, several layout types were developed and compared to find the optimal topology.

\section{B. Layout}

To perform the antenna layout, the following design procedure is adopted. The first is to determine the operation frequency, the electrical length, and characteristic impedance of the RH and LH transmission lines. Here, the operation frequency is $2.45 \mathrm{GHz}$ and the characteristic impedance $\left(\mathrm{Z}_{0}\right)$ of the LH and RH TLs is set to be $25 \mathrm{Ohm}$. The second is using the circuit model to determine the values of required inductors and capacitors. This can be done by applying Table I. It turns out that all the three capacitors have the same value of 2.6 $\mathrm{pF}$ and both of the inductors have the same value of $1.62 \mathrm{nH}$ at $2.45 \mathrm{GHz}$. The third, the most important step, is to realize these capacitors and inductors by using printed elements. The capacitors are patches against the ground with proper size to fit the value of capacitance. The equation of the parallel plate capacitor can be used to get the initial sizes of the patches. The inductors are implemented by metal traces or metal traces with slotted ground. The lengths of metal traces and the sizes of the slots are designed by EM simulator to fit the value of inductance. Finally, the obtained printed capacitors and inductors are connected properly according to the circuit model. The arrangement of these elements is important for antenna

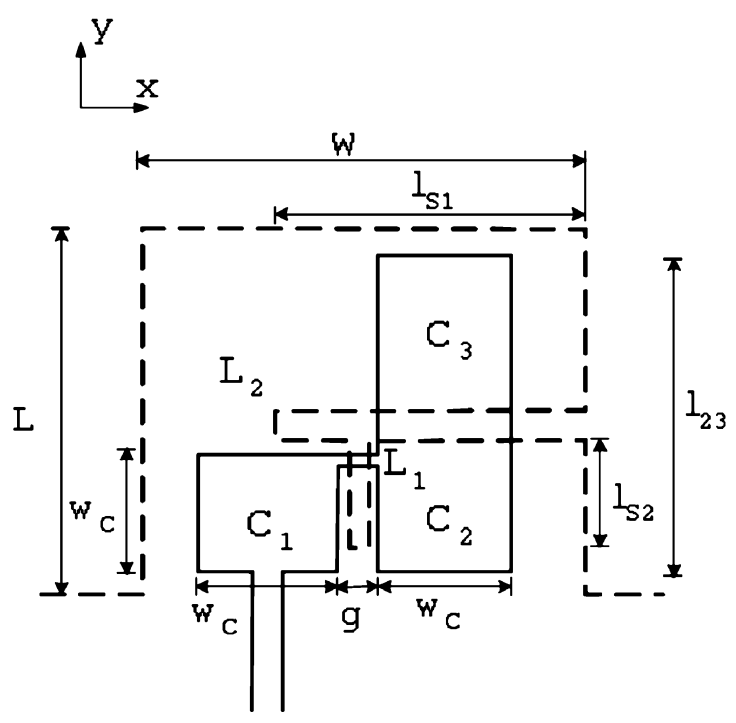

Fig. 6. Layout of the proposed antenna. The dashed lines are for the bottom metal layout and the solid lines are for the top metal layout.

performance. The layout consideration becomes the point of this antenna design. For planning the layouts, full wave EM simulation is done by utilizing the commercial software, Ansoft HFSS. Three possible layouts are considered for comparison as follows. The layout is made on a two sided FR4 substrate with relative dielectric constant of 4.4 and thickness of $0.4 \mathrm{~mm}$.

A compact antenna structure based on the circuit in Fig. 5 is proposed as illustrated in Fig. 6 with the corresponding elements marked. The solid lines are for the top metal layout and the dashed lines are for the bottom layout. The rectangular patches are obviously planned to realize the capacitors. The narrow short metal traces dominantly contribute as inductors. This layout arranges the $\mathrm{C}_{1}, \mathrm{~L}_{1}, \mathrm{C}_{2}, \mathrm{C}_{3}$, and $\mathrm{L}_{2}$ in turn forms a closed loop and intend to create the longer slot on the ground plane. The geometry parameters are $L=W=11.5 \mathrm{~mm}, w_{C}=4 \mathrm{~mm}$, $l_{23}=9.5 \mathrm{~mm}, g=1.3 \mathrm{~mm}, l_{\mathrm{S} 1}=7.2 \mathrm{~mm}, l_{\mathrm{S} 2}=3.2 \mathrm{~mm}$. Instead of a narrow short trace, the small inductor $\mathrm{L}_{2}$ is realized by a wide and longer metal trace. Besides, a slot of width of $0.5 \mathrm{~mm}$ under $\mathrm{L}_{1}$ is introduced and observed to provide a wide tuning range for the input resistance by changing the slot length $l_{\mathrm{S} 2}$.

Two other possible layouts, named the type A and B, are shown in Fig. 7(a) and (b), which are made also on the same FR4 substrate. For the type A layout, the bottom pattern is a wide trace of $5.5 \mathrm{~mm}$ in width with, of course, a narrow trace for $\mathrm{L}_{2}$. The top pattern is a less wide trace of $3.5 \mathrm{~mm}$ in width also with a narrow trace of $2.5 \mathrm{~mm}$ in length for $\mathrm{L}_{1}$. The type B layout has quite similar dimension with the type A layout but its wide traces go along an edge of the ground plane. The trace for $\mathrm{L}_{2}$ is directly connected to the ground plane. The dimension along the $y$ axis of the straight type A antenna is $18.5 \mathrm{~mm}$ and the dimension along the $x$ axis of the straight type $\mathrm{B}$ antenna is $18 \mathrm{~mm}$. It can be roughly interpreted that the proposed layout is obtained by bending the straighter layout as the type A or B. It should be mentioned that all the three layouts have finite ground planes of $40 \mathrm{~mm}$ ( $y$-direction) by $30 \mathrm{~mm}$ ( $x$-direction) in size, 


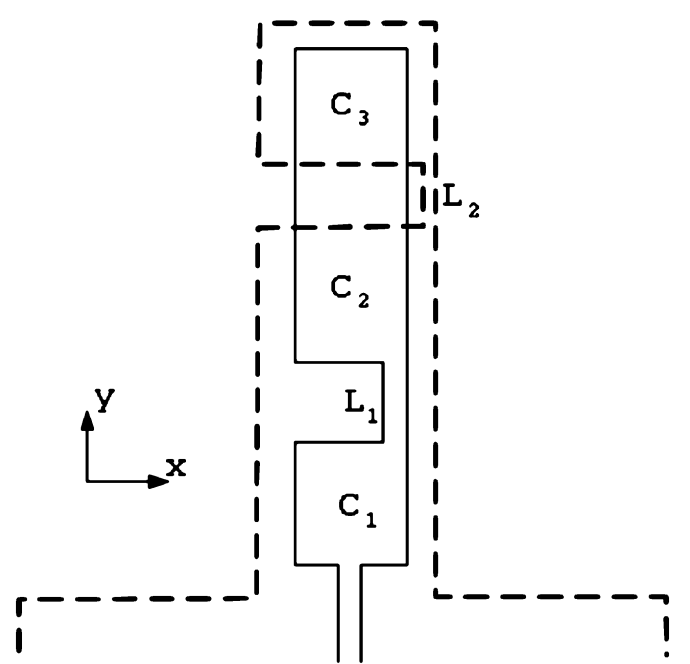

(a)

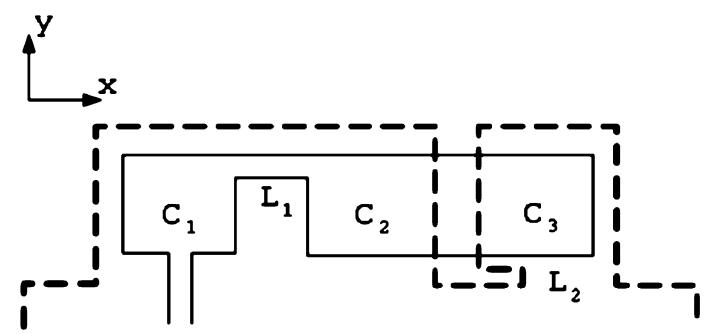

(b)

Fig. 7. Layouts of (a) straight type A antenna and (b) straight type B antenna. The dashed lines are for the bottom metal layout and the solid lines are for the top metal layout.

extended in the negative $y$ direction and partly ignored in the figures for simplicity of illustration.

\section{RADIATION MECHANISM}

Different layouts may cause different electric field and current distributions, thus affecting the radiation performance. Fig. 8 shows the simulated input impedances of all the three antenna layouts. At the design frequency of $2.45 \mathrm{GHz}$, all of them possess an anti-resonance, (or a parallel resonance) as can be observed from Fig. 8(b) where the curve for the input reactance have a zero crossing with a negative slope at that frequency. However, apparent differences of the real parts are observed. The real part of antenna input impedance usually reflects the radiation performance for easy matching with good radiation efficiency. Thus the layout planning has significant influences on providing efficient radiating structures while employing the cascaded right/left-handed transmission lines.

It is observed that a second anti-resonance occurs around the harmonic frequency $(4.5 \mathrm{GHz})$ for both the type $\mathrm{A}$ and $\mathrm{B}$ antennas with straight layout. This second anti-resonance also happens in the proposed antenna, however, at a lower frequency of $3.5 \mathrm{GHz}$. Since the design and analysis focus on the performance at the desired fundamental frequency, other resonances are not taken into account in this paper.

The most difference between these three layouts is considered as the topology of the ground. The proposed antenna structure

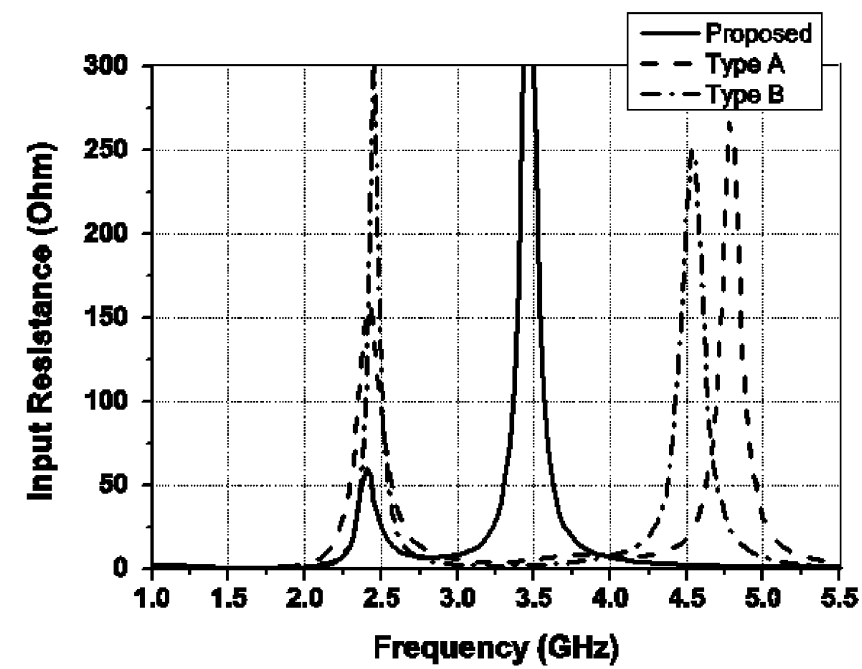

(a)

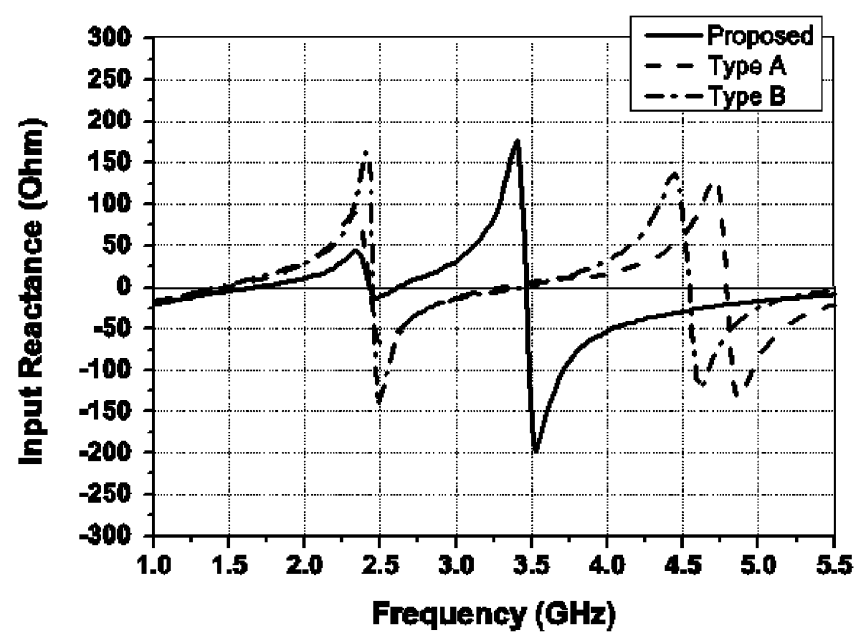

(b)

Fig. 8. Simulated (a) input resistance and (b) reactance for the proposed, type A, and type B antennas.

can be interpreted as closing the layout for $\mathrm{L}_{2}$ to the bottom side of $\mathrm{C}_{1}$. The surface electric current flow is guided roughly in a loop in the order of $\mathrm{C}_{1}, \mathrm{~L}_{1}, \mathrm{C}_{2}, \mathrm{C}_{3}, \mathrm{~L}_{2}$ and then back to $\mathrm{C}_{1}$. In contrast, the type $\mathrm{A}$ and $\mathrm{B}$ is extended straightly without closing the ground plane to the bottom side of $\mathrm{C}_{1}$. This difference causes the proposed layout introduces longer slot on the ground plane which may have significant influences on inducing radiating current on the ground efficiently. It is found that the slot length, $l_{\mathrm{S} 2}$ of the proposed layout, can be used to reduce the input resistance of the antenna from hundreds of ohms to a few ohms with unapparent changing of resonant frequency shown in Fig. 9. It proves the influence of the radiation from the proper slot on the ground plane. In addition, the proposed antenna can be easily matched.

Besides the consideration of the slot, the patches as capacitors which occupy the main area of the antenna are also studied. An individual patch of small size with respect to the wavelength can only generate negligible far field radiation since the equivalent surface magnetic current $\left(\mathbf{M}_{\mathbf{S}}\right)$ at the edges results in canceling 


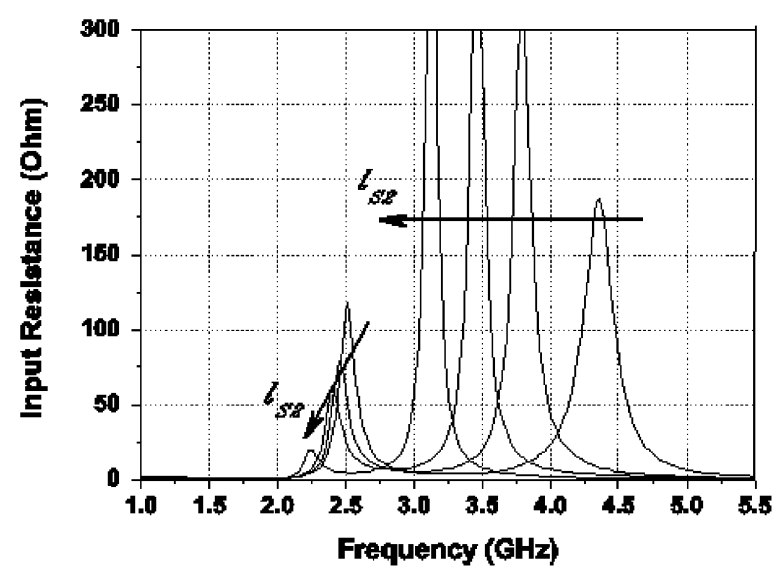

(a)

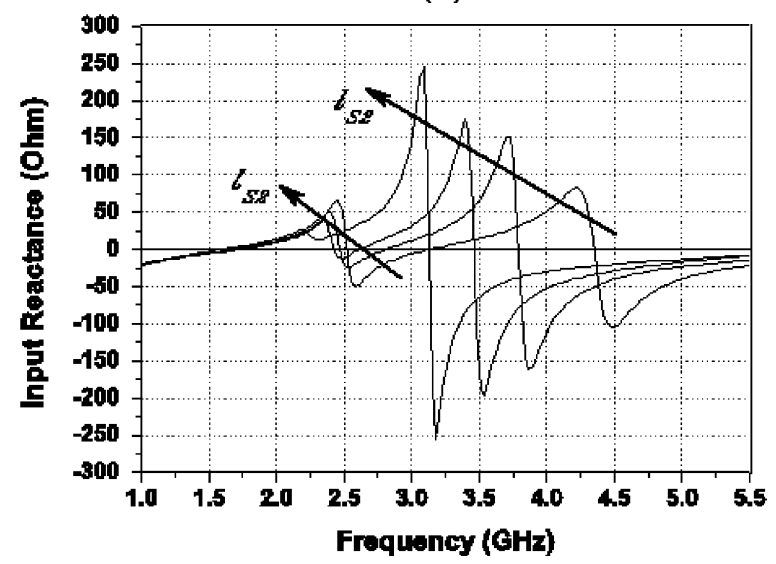

(b)

Fig. 9. Simulated (a) real part and (b) imaginary part of the input impedance, with changing the slot length $l_{\mathrm{S} 2}$ under $\mathrm{L}_{1}$, for the proposed antenna. $l_{\mathrm{S} 2}=$ $1.5,3.0,3.5,4.5 \mathrm{~mm}$.

each other at bore sight direction. A surface magnetic current $\mathbf{M}_{\mathbf{S}}$ is defined by

$$
\mathbf{M}_{\mathbf{S}}=\mathbf{E} \times \hat{\mathbf{n}}
$$

where $\mathbf{E}$ is the electric field, and $\hat{\mathbf{n}}$ is the outward normal of the side walls for capacitor patches or the normal vector to the surface of the slots. The proposed layout (Fig. 6) has a topology of patches at the top and slots at the bottom. The bottom pattern could be considered as the extended ground with two connected orthogonal slots. The capacitors confine electric energy and provide fringing fields at their edges. As seen in Fig. 10(a), the simulated electric fields for $\mathrm{C}_{2}$ and $\mathrm{C}_{3}$ possess opposite polarities, i.e., the electric field vectors for $\mathrm{C}_{2}$ points upward if those for $\mathrm{C}_{3}$ points downward. The opposite polarities make the equivalent magnetic current flow from $\mathrm{C}_{2}$ goes counterclockwise while that from $\mathrm{C}_{3}$ goes clockwise, as indicated by the double-headed arrows of Fig. 10(b). The radiation fields from the close and opposite directed pairs of the magnetic currents cancel with each other. Therefore, as a result, there are two edges at the top constructively contributing to radiation. These two edges provided by $\mathrm{C}_{2}$ and $\mathrm{C}_{3}$ at the top side operate as

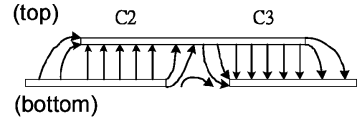

(a)

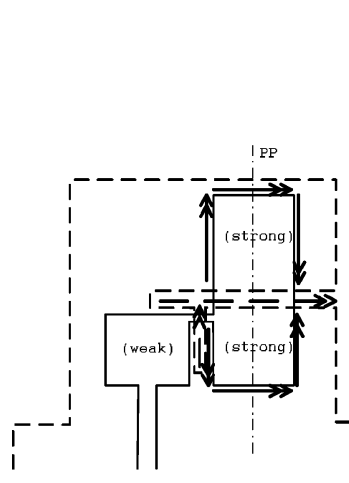

(b)

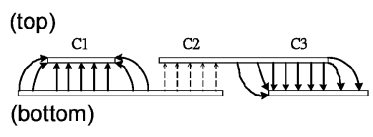

(c)

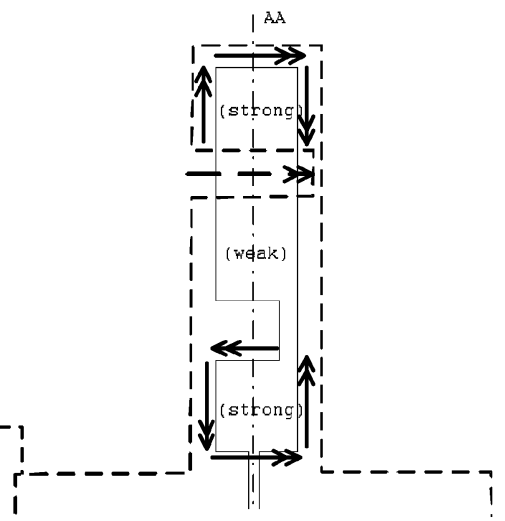

(d)

Fig. 10. (a) Schematic for the electric field distribution at the PP cut for the proposed antenna. (b) Schematic of the equivalent magnetic current distribution for the proposed antenna. (c) Schematic of the electric field distribution at the AA cut for the type A antenna. (d) Schematic of the equivalent magnetic current distribution for the type A antenna.

the radiating edges of a conventional half-wavelength patch antenna. In other word, this design shrinks the dimension from half wavelength to one-eleven wavelength. Moreover, there are two connected slots at the bottom side offering aperture electric field for constructing radiation. The contribution provided by $\mathrm{C}_{1}$ is not taken into account since its field strength is very weak.

Regarding the type A, it plans three patches in a row with two short traces. The corresponding simulated electric fields and equivalent magnetic currents are shown in Fig. 10(c) and (d), respectively. The magnetic currents provided by $\mathrm{C}_{1}$ cancel each other in the far field since they are close and opposite directed in pairs. The fringing field from $\mathrm{C}_{2}$ is too weak to contribute. Two remaining edges provide equivalent magnetic currents in the same direction. One is from the slot at the bottom side between $\mathrm{C}_{2}$ and $\mathrm{C}_{3}$. The other one is the upper edge of $\mathrm{C}_{3}$, which is close and parallel to the slot one. The type $\mathrm{B}$ has very similar field distribution with the type A.

\section{EXPERIMENTS}

For experimental verification, both the type A and the proposed structure are fabricated and measured. These two printed antennas were implemented on an FR4 substrate with relative dielectric constant of 4.4 and thickness of $0.4 \mathrm{~mm}$. The proposed antenna results in occupying an area of $11.5 \mathrm{~mm}$ by $11.5 \mathrm{~mm}$ with a connected ground size of $40 \mathrm{~mm}$ by $30 \mathrm{~mm}$. The type A has the size of $5.5 \mathrm{~mm}$ by $18.5 \mathrm{~mm}$ with the same ground size as the proposed one.

As the simulation result predicted, the input resistance of the type A antenna is about $150 \Omega$, which is relatively large for the 50- $\Omega$ system. Thus, an extra quarter-wavelength high impedance line is added between $C_{1}$ and the 50- $\Omega$ microstrip feeding line for impedance transformation. Fig. 11 shows the measured and simulated return losses, as functions of frequency, for the proposed antenna and the type A antenna. Both of the 


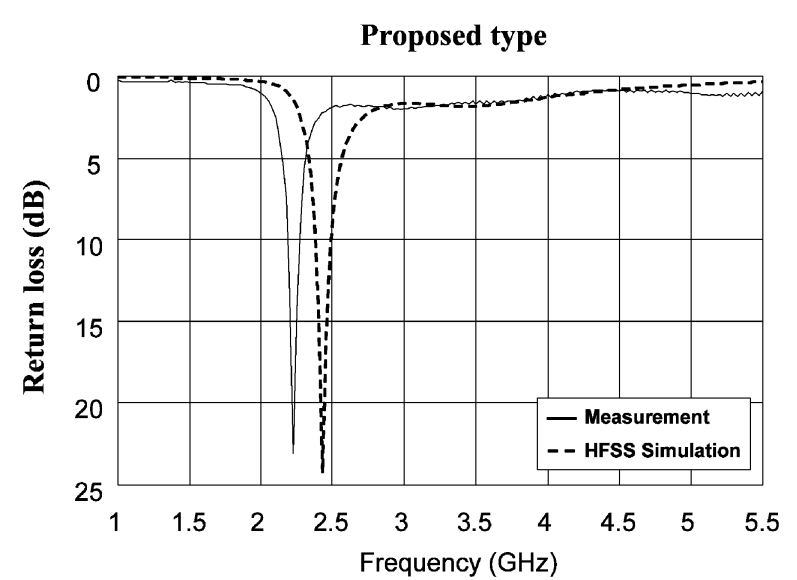

(a)

Type A

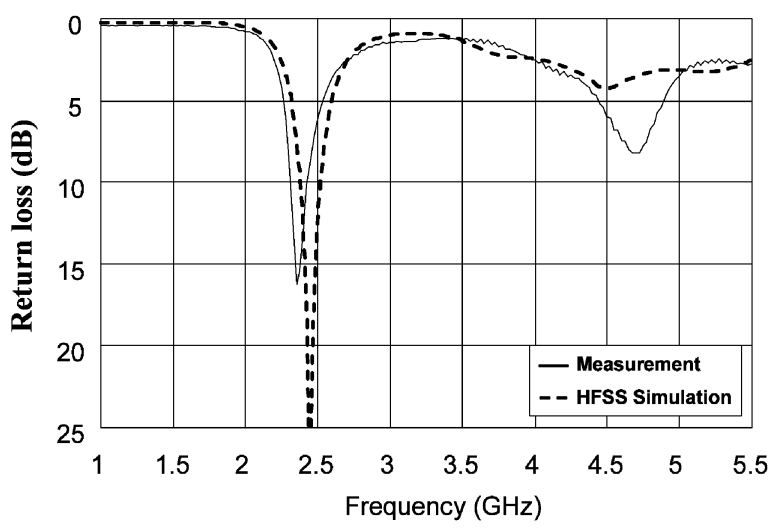

(b)

Fig. 11. Simulated and measured return loss for (a) the proposed antenna and (b) the type A antenna.

measurement results shift a little in frequency from the desired $2.45 \mathrm{GHz}$. The proposed antenna exhibits a resonant frequency at $2.23 \mathrm{GHz}$ with the measured return loss of $23 \mathrm{~dB}$, whereas the type A antenna is at $2.35 \mathrm{GHz}$ with a return loss of $16 \mathrm{~dB}$. The corresponding 10-dB return loss bandwidths are $4.5 \%$ and $5.3 \%$, respectively.

Figs. 12 and 13 show the measured radiation patterns for the proposed antenna and the type A antenna, respectively, in the three principal planes. The peak gain is $+0.16 \mathrm{dBi}$ for the proposed antenna and is $-0.54 \mathrm{dBi}$ for the type A antenna.

To further understand the influence of the small patches on the radiation performance, two antennas, which have the same layout as the proposed antenna but with the printed capacitors in one antenna replaced by lumped capacitors [Fig. 14(a) and (b)], were designed and measured for comparison. Both antennas use same ground plane design with the same slots and equivalent capacitors and inductors. The measurement results and the photos of the antennas are shown in Fig. 14. The input resistance of the antenna with lumped capacitor is a little less than the other at the center frequency, as can be observed from Fig. 14(c) and (d). Also from Fig. 14(e) and (f), it is seen that, the radiation patterns of the two antennas are quite similar, which means that the capacitor patches do not contribute significant radiation. Most of
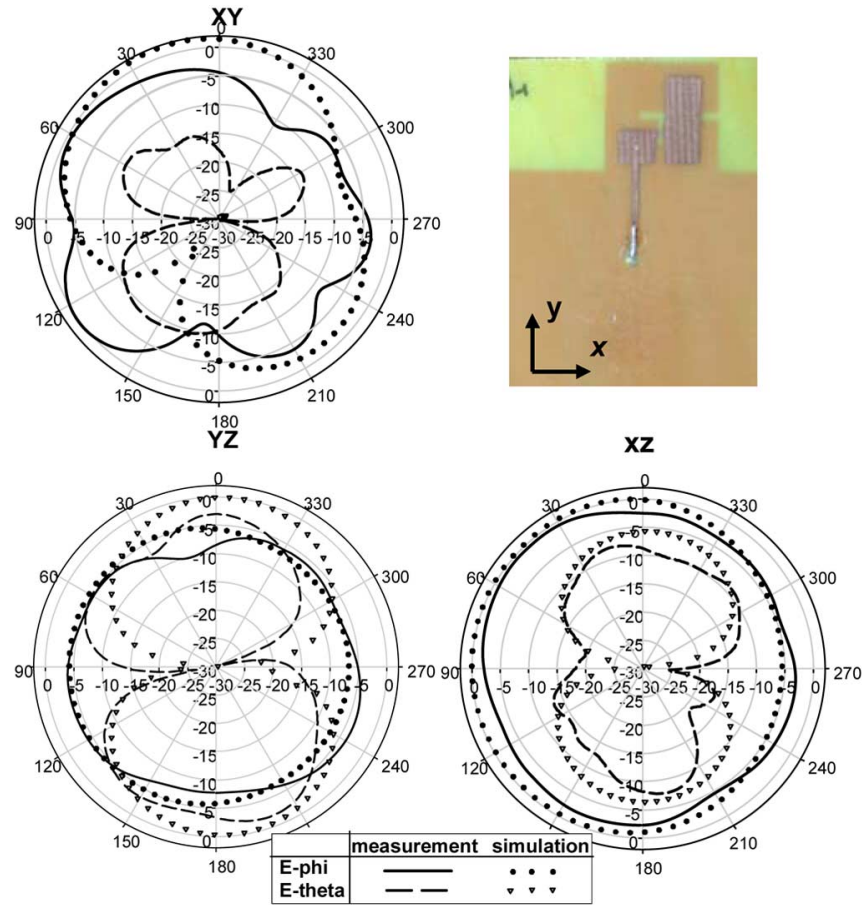

Fig. 12. Simulated and measured radiation patterns for the proposed antenna.
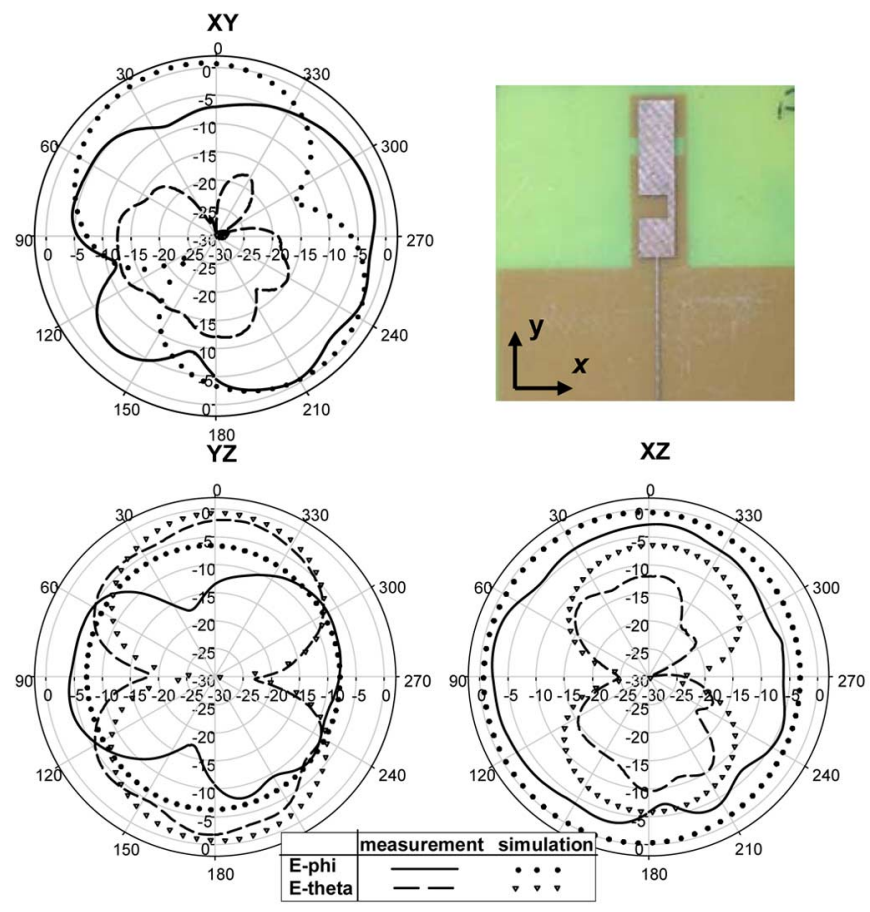

Fig. 13. Simulated and measured radiation patterns for the type-A antenna.

the radiation comes from the ground slots and the induced currents along the ground edges. Nevertheless, it is still noticeable that the theta component of the electrical field of the antenna with patch capacitors is about $2 \mathrm{~dB}$ larger than that of the antenna with lumped capacitors.

Through careful design and layout planning, the proposed antenna physically formed by small patches and slots can offer 


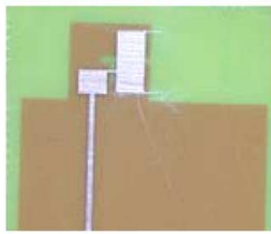

(a)

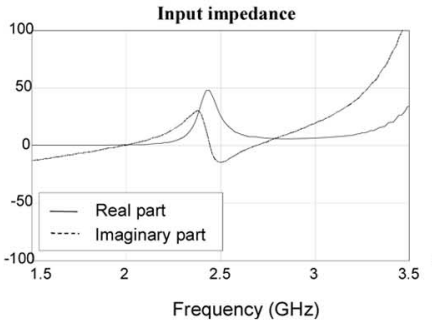

(c)

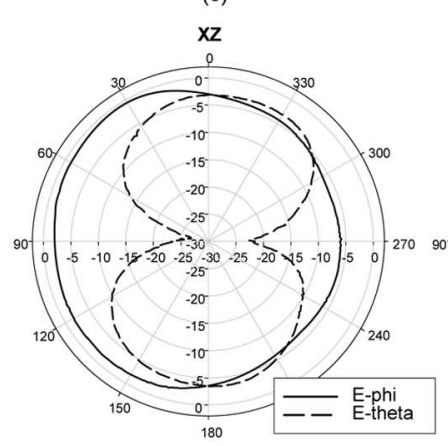

(e)

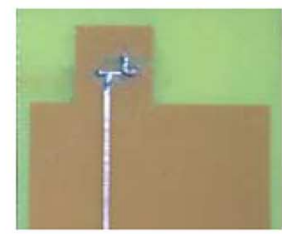

(b)

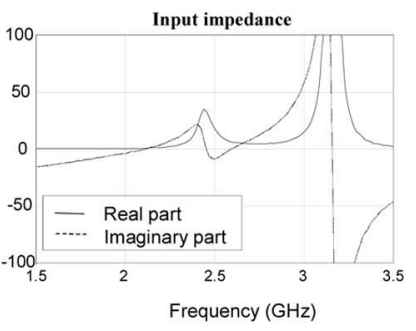

(d)

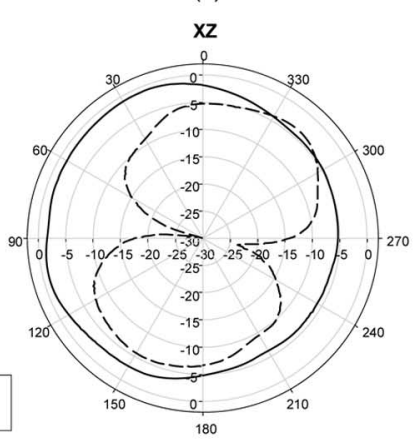

(f)
Fig. 14. (a) The realized proposed antenna layout with printed elements. (b) The realized proposed antenna layout with lumped capacitors. (c) The measured input impedance of the antenna with printed element. (d) The measured input impedance of the antenna with lumped capacitors. (e) The measured radiation pattern of the antenna with printed element in $\mathrm{X}-\mathrm{Z}$ plane. (f) The measured radiation pattern of the antenna with lumped capacitors in $\mathrm{x}-\mathrm{Z}$ plane.

fairly good performance with a compact dimension of $\lambda_{0} / 11$ square, where $\lambda_{0}$ is the free space wavelength.

Finally, due to the self-resonance of the antenna configuration, the resonant frequency of the proposed antenna is insensitive to the ground size, as can be observed from the measurement results shown in Fig. 15. Here, three antennas with the ground sizes of $70 \mathrm{~mm}$ by $45 \mathrm{~mm}, 40 \mathrm{~mm}$ by $20 \mathrm{~mm}$, and $27 \mathrm{~mm}$ by $25 \mathrm{~mm}$ using same antenna layout are measured and compared. It is seen that the resonant frequencies of the three antennas are almost the same. It proves that the ground size has negligible effect on the resonance of the proposed antenna structure. As a reference, three typical printed quarter-wavelength monopole antenna with different ground sizes have also been developed for comparison. The antennas have the same monopole strip size of $27 \mathrm{~mm}$ by $1.6 \mathrm{~mm}$ and with ground sizes of $70 \mathrm{~mm}$ by $45 \mathrm{~mm}$, $40 \mathrm{~mm}$ by $20 \mathrm{~mm}$, and $27 \mathrm{~mm}$ by $25 \mathrm{~mm}$, respectively. Although not shown here, the center frequency of the monopole antenna varies with the change of the ground size from 2.35 to $1.99 \mathrm{GHz}$. A $16.5 \%$ frequency offset is observed.

\section{CONCLUSION}

A novel compact planar antenna is designed and verified experimentally. Fairly good measurement results are obtained.
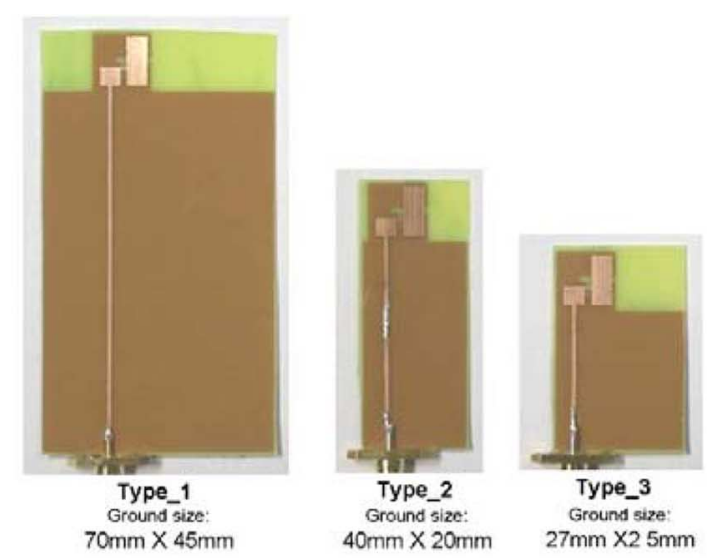

(a)

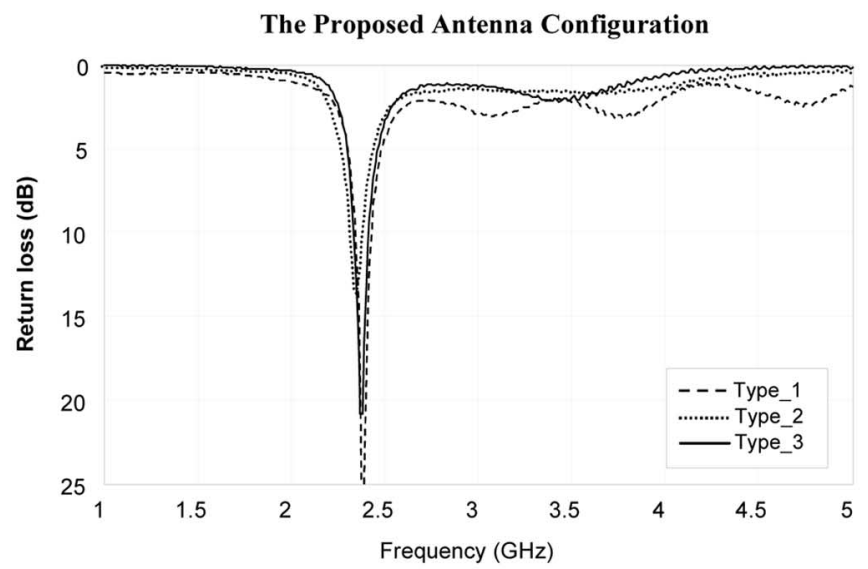

(b)

Fig. 15. (a) The proposed antenna configuration with different ground sizes. (b) The return loss of the proposed antenna configuration with different ground sizes.

Layout planning plays a crucial role for radiating as discussed. Different layouts for the same circuit model can cause different performances. This paper shows the possibility to design compact antennas based on cascaded right/left-handed transmission lines. Through applying the equivalent transmission line model, the physical dimension can be compact with a size are small as $\lambda_{0} / 11$ square. In this paper, the proposed antenna avoids via and consists of only five lumped elements by the selection of the EQC model combination. The $\pi$ and $\mathrm{T}$ models can offer exact formulas for $\mathrm{L}$ and $\mathrm{C}$ for almost all-range electrical length, $\theta$, rather than CRLH TL of which formulas for the circuit elements are valid for very small $\theta$ with the same accuracy as $\sin \theta$ approaches unity. (In fact, the CRLH TL method can be considered as a sub-category of the general $\pi$ and T models.) Possible radiation mechanism, including the radiation from the capacitor patches and the slots, of the proposed antenna has also been proposed. After the experimental comparison as shown in Fig. 14, it is found that the antenna is more like a slot radiator. Instead of only half-space patch-like radiation, the proposed antenna structure with more than two radiating edges gives a fairly omnidirectional radiation pattern. The proposed design has several advantages, such as easily achieving resonance, simple structure, compact size without performance degradation, and stable frequency against different ground size. 
Additionally, although the EQC parameters in this paper are chosen for certain considerations, different electrical length, different characteristic impedance, or different equivalent models are still possible to be applied.

\section{ACKNOWLEDGMENT}

The authors would like to give their deep appreciation to the reviewers for the valuable discussion about the antenna radiation mechanism, which makes the paper more solid.

\section{REFERENCES}

[1] L. Liu, C. Caloz, and T. Itoh, "Dominant mode leaky-wave antenna with backfire-to-endfire scanning capability," Elect. Lett., vol. 38, pp. 1414-1416, Nov. 2002.

[2] S. Lim, C. Caloz, and T. Itoh, "A reflectodirective system using a composite right/left-handed (CRLH) leaky-wave antenna and heterodyne mixing," IEEE Microw. Wireless Compon. Lett., vol. 14, no. 4, pp. 183-185, Apr. 2004.

[3] C. A. Allen, C. Caloz, and T. Itoh, "Leaky-waves in a metamaterialbased two-dimensional structure for a conical beam antenna application," in IEEE MTT-S Int. Microwave Symp. Dig., Jun. 2004, vol. 1, pp. 305-308.

[4] C. Caloz and T. Itoh, "Array factor approach of leaky-wave antennas and application to 1-D/2-D composite right/left-handed (CRLH) structures," IEEE Microw. Wireless Compon. Lett., vol. 14, pp. 274-276, Jun. 2004.

[5] S. Lim, C. Caloz, and T. Itoh, "Electronically scanned composite right/ left handed microstrip leaky-wave antenna," IEEE Microw. Wireless Compon. Lett., vol. 14, no. 6, pp. 277-279, Jun. 2004.

[6] S. Lim, C. Caloz, and T. Itoh, "Metamaterial-based electronically controlled transmission-line structure as a novel leaky-wave antenna with tunable radiation angle and beamwidth," IEEE Trans. Microw. Theory Tech., vol. 53, no. 1, pp. 161-173, Jan. 2005.

[7] S. Matsuzawa, K. Sato, A. Sanada, H. Kubo, and S. Aso, "Left-handed leaky wave antenna for millimeter-wave applications," in Proc. IEEE Int. Workshop on Antenna Technology Small Antennas and Novel Metamaterials, Mar. 2005, pp. 183-186.

[8] C. A. Allen, K. M. K. H. Leong, C. Caloz, and T. Itoh, "A two-dimensional edge excited metamaterial-based leaky wave antenna," in Proc. IEEE Antennas Propag. Soc. Int. Symp., Jul. 2005, vol. 2B, pp. 320-323.

[9] K. Sato, S. Matsuzawa, Y. Inoue, and T. Nomura, "Electronically scanned left-handed leaky wave antenna for millimeter-wave automotive applications," in Proc. IEEE Int. Workshop Antenna Technology Small Antennas and Novel Metamaterials, Mar. 2006, pp. 420-423.

[10] M. Schussler, J. Freese, and R. Jakoby, "Design of compact planar antennas using LH-transmission lines," in IEEE MTT-S Int. Microwave Symp. Dig., Jun. 2004, vol. 1, pp. 6-11.

[11] A. Sanada, K. Murakami, S. Aso, H. Kubo, and I. Awai, "A via-free microstrip left-handed transmission line," in IEEE MTT-S Int. Microwave Symp. Dig., Jun. 2004, vol. 1, pp. 301-304.

[12] A. Sanada, M. Kimura, I. Awai, C. Caloz, and T. Itoh, "A planar zerothorder resonator antenna using a left-handed transmission line," in Proc. Eur. Microwave Conf., Amsterdam, The Netherlands, Oct. 2004, pp. 1341-1344.

[13] C.-J. Lee, K. M. K. H. Leong, and T. Itoh, "Design of resonant small antenna using composite right/left-handed transmission line," in Proc. IEEE Antenna Propag. Soc. Int. Symp., Jul. 2005, vol. 2B, pp. 218-221.

[14] A. Lai, K. M. K. H. Leong, and T. Itoh, "Dual-mode compact microstrip antenna based on fundamental backward wave," in Proc. AsiaPacific Microwave Conf., Suzhou, China, Dec. 2005, vol. 4.

[15] S. Otto, A. Rennings, C. Caloz, P. Waldow, and T. Itoh, "Composite right/left-handed lambda-resonator ring antenna for dual-frequency operation," in Proc. IEEE Antennas Propag. Soc. Int. Symp., Jul. 2005, vol. 1A, pp. 684-687.

[16] M. Schussler, C. Damm, J. Freese, and R. Jakoby, "Realization concepts for compact microstrip antennas with periodically loaded lines," in IEEE MTT-S Int. Microwave Symp. Dig., Jun. 12-17, 2005, p. 4.
[17] Z. Qi and L. Huan, "Design of printed antenna with left-handed array," in Proc. Asia-Pacific Microwave Conf., Suzhou, China, Dec. 2005, vol. 4.

[18] A. Lai, K. M. K. H. Leong, and T. Itoh, "Composite right/left-handed metamaterial antennas," in Proc. IEEE Int. Workshop on Antenna Technology Small Antennas and Novel Metamaterials, Mar. 6-8, 2006, pp. 404-407.

[19] F. Qureshi, M. A. Antoniades, and G. V. Eleftheriades, "A compact and low-profile metamaterial ring antenna with vertical polarization," Antennas Wireless Propag. Lett., vol. 4, pp. 333-336, 2005.

[20] C.-J. Lee, K. M. K. H. Leong, and T. Itoh, "A broadband microstrip-to-CPS transition using composite right/left-handed transmission lines with an antenna application," presented at the IEEE MTT-S Int. Microwave Symp. Dig., Long Beach, CA, Jun. 12-17, 2005.

[21] K. Z. Rajab, R. Mittra, and M. T. Lanagan, "Size reduction of microstrip antennas using metamaterials," in Proc. IEEE Antennas Propag. Soc. Int. Symp., Jul. 2005, vol. 2B, pp. 296-299.

[22] A. Lai, K. M. K. H. Leong, and T. Itoh, "Novel series divider for antenna arrays with arbitrary element spacing based on a composite right/left-handed transmission line," in Proc. Eur. Microwave Conf., Oct. 2005, vol. 1, pp. 145-148.

[23] Z. Zhang and S. Xu, "A novel feeding network with composite right/ left-handed transmission line for 2-dimension millimeter wave patch arrays," in Proc. Asia-Pacific Microwave Conf., Suzhou, China, Dec. 2005, vol. 3 .

[24] S.-G. Mao and Y.-Z. Chueh, "Broadband composite right/left-handed coplanar waveguide power splitters with arbitrary phase responses and balun and antenna applications," IEEE Trans. Antennas Propag., vol. 54, pp. 234-250, Jan. 2006.

[25] H. Iizuka and P. S. Hall, "A left-handed dipole concept," in Proc. IEEE Int. Workshop on Antenna Technology Small Antennas and Novel Metamaterials, Mar. 2006, pp. 396-399.

[26] S.-G. Mao and S.-L. Chen, "Characterization and modeling of lefthanded microstrip lines with application to loop antennas," IEEE Trans. Antennas Propag., vol. 54, no. 4, pp. 1084-1091, Apr. 2006.

[27] C. Caloz and T. Itoh, "Novel microwave devices and structures based on the transmission line approach of meta-materials," in IEEE MTT-S Int. Microwave Symp. Dig., Jun. 2003, pp. 195-198.

[28] A. Sanada, C. Caloz, and T. Itoh, "Novel zeroth-order resonance in composite right/left-handed transmission line resonators," in Proc. Asia-Pacific Microwave Conf., Seoul, Korea, Nov. 2003, pp. $1588-1592$.

[29] S. Ramo, J. R. Whinnery, and T. Van Duzer, Fields and Waves in Communication Electronics, 3rd ed. New York: Wiley, 1994, ch. 5.

[30] D. Kuylenstierna, S. E. Gunnarsson, and H. Zirath, "Lumped-element quadrature power splitters using mixed right/left-handed transmission lines," IEEE Trans. Microw. Theory Tech., vol. 53, no. 8, pp. 2616-2621, Aug. 2005.

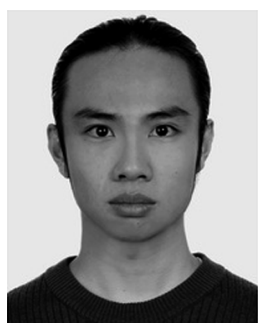

Yu-Shin Wang was born in Taichung, Taiwan, R.O.C., in May 1981. He received the B.S. degree in communication engineering from the National Chiao Tung University, Hsinchu, Taiwan, R.O.C., in 2003, where he is currently working toward the $\mathrm{Ph} . \mathrm{D}$. degree in communication engineering.

$\mathrm{He}$ is currently involved with research on microwave circuits, antennas and antenna arrays.

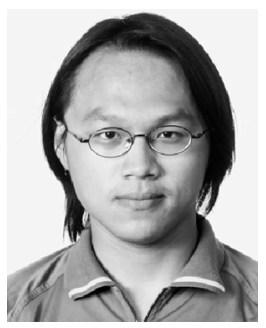

Min-Feng Hsu was born in Hsinchu, Taiwan, R.O.C., on November 21, 1981. He received the B.S. degree in communication engineering from National Chiao Tung University, Hsinchu, Taiwan, R.O.C., in 2004, where he is currently working toward the M.S. degree in communication engineering.

His main research interests are microwave circuits, planar antenna design and metamaterial. 


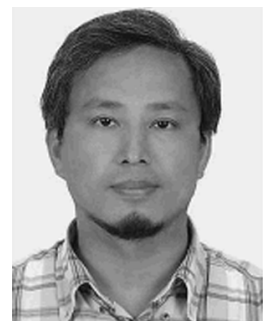

Shyh-Jong Chung (M'92-SM'06) was born in Taipei, Taiwan, R.O.C. He received the B.S.E.E. and $\mathrm{Ph} . \mathrm{D}$. degrees from National Taiwan University, Taipei, Taiwan, R.O.C., in 1984 and 1988, respectively.

Since 1988, he has been with the Department of Communication Engineering, National Chiao Tung University, Hsinchu, Taiwan, R.O.C., where he is currently a Professor. From September 1995 to August 1996, he was a Visiting Scholar with the Department of Electrical Engineering, Texas, A\&M University,
College Station. He has authored or coauthored over 70 technical papers in international journals or conferences including several invited papers and speeches His areas of interest include the design and applications of active and passive planar antennas, communications in intelligent transportation systems (ITSs), LTCC-based RF components and modules, packaging effects of microwave circuits, and numerical techniques in electromagnetics.

Dr. Chung has served as Chairman of the IEEE MTT-S Taipei Chapter since 2005. He was also the Treasurer of IEEE Taipei Section from 2001 to 2003. 